\section{Educación para el desarrollo o la promesa de empleabilidad como factor de formación}

\author{
Education for Development or \\ the Promise of Employability \\ as a Formation Factor
}
Educação para o desenvolvimento ou a promessa de empregabilidade como um fator de formação

\section{Hedilberto Granados López*}

\section{Resumen}

Inscrito en el horizonte de articulación entre educación y desarrollo, el artículo muestra algunos de los resultados de un trabajo investigativo en el que se recogen conceptualizaciones de profesionales vinculados a la educación de diversos municipios del Departamento de Caldas. Las categorías utilizadas para revisar las conceptualizaciones permiten poner en evidencia que si bien prevalece una mirada romántica asociada a la educación como base para el desarrollo humano, esta se encuentra supeditada a la posibilidad que tenga lo educativo de impactar significativamente el desarrollo económico individual y comunitario.

\section{Palabras clave}

Educación para el desarrollo, bienestar social, empleabilidad, desarrollo humano

\section{Abstract}

This article, dealing with the articulation between education and development, shows some of the results of a research which includes conceptualizations of education professionals from various municipalities in the Department of Caldas. The categories used to review the conceptualizations reveal that although a romantic look associated with education prevails as a basis for human development, it is subject to the potential of education to impact significantly both the individual and community economic development.

\section{Key words}

Education for development, social welfare, employability, human development

\section{Resumo}

Inscrito no horizonte de articulação entre educação e desenvolvimento, o artigo mostra alguns dos resultados de um trabalho de pesquisa que inclui conceituações de profissionais ligados à educação de vários municípios do Departamento de Caldas. As categorias usadas para rever os conceitos permitem colocar em evidência que, embora prevaleça um olhar romântico associado a educação como base para o desenvolvimento humano, esta se encontra subordinada à possibilidade que a educação tem de impactar significativamente o desenvolvimento econômico do indivíduo e da comunidade.

\section{Palavras chave}

Educação para o desenvolvimento, bem-estar social, empregabilidade, desenvolvimento humano

Fecha de recepción: Mayo 8 de 2015

Fecha de aprobación: Septiembre 17 de 2015 


\section{Introducción}

$\mathrm{E}$ l desarrollo como estrategia histórica sugiere el despliegue de una serie de articulaciones con distintos actores sociales, que permita de una manera directa no solo fijar unos objetivos determinados, sino también una vigilancia sobre su cumplimiento y mejora. Dentro de las estrategias que se han desprendido del desarrollo como estrategia histórica, se encuentra la premisa vigente hasta nuestros días, en la cual, el sector educativo representa y constituye uno de los pilares para la transformación de la economía social y en consecuencia del desarrollo comunitario.

Esta premisa sobre lo educativo representa una buena oportunidad para valorar los avances y logros que la educación como estrategia del desarrollo ha tenido en el despliegue y transformación de estructuras sociales. De igual manera, permite una valoración adicional, al poder evaluar los avances que el sector educativo ha tenido, desde una lógica reproductiva de factores e indicadores económicos, a una concepción más cercana a lo humano y el bienestar social.

En consecuencia, el presente documento mostrará, a partir de los datos obtenidos en un estudio de tipo descriptivo en el departamento de Caldas, el uso/frecuencia de algunas de las concepciones que sobre lo educativo prevalecen en asociación a un determinado uso nominativo del término, y en correlación a una mirada sobre el desarrollo de acuerdo con la presencia de un determinado uso/frecuencia de categorías alusivas al desarrollo.

El estudio resulta significativo, toda vez que pueda dar cuenta o no de un avance social significativo según el uso nominativo que se hace de lo educativo como valor comunitario y eje de transformación social, más allá de la perspectiva histórica de la educación y su maridaje con las posturas económicas del desarrollo para el denominado cambio social.

\section{Perspectivas históricas sobre una educación para el desarrollo}

La idea de desarrollo en el campo educativo -o lo que podría entenderse como la instrumentalización de lo educativo como un dispositivo de mediación cultural desde la perspectiva economicista- tiene por antecedentes las consignas del presidente Harry Truman en 1949, y que darían inicio a toda una dinámica de transformación de los imaginarios sociales (Castoriadis, 1997) pensados para solventar el déficit económico dejado por la Segunda Guerra Mundial y que serviría de complemento al denominado plan Marshall de 1947.

De acuerdo con Prats (2010), en la construcción histórica de la idea de desarrollo, "la matriz intelectual del desarrollo se encuentra en el conjunto de ideas y creencias articuladas al llamado racionalismo constructivista, y al racionalismo evolutivo crítico" (p. 3).

En consecuencia, y de acuerdo con lo que podría leerse sobre los inicios de la idea de desarrollo es cierto tipo de perspectiva racionalista de carácter universalista e instrumental, de la cual forma parte inherente lo educativo (Prats, 2010) y que da pie a la intervención del Estado y otros agentes de orden económico en la planificación como un valor fundamental para llevar a cabo las premisas del desarrollo en el ámbito educativo.

Así pues, la planificación como estrategia o disciplina que da piso científico a la nueva mirada sobre el desarrollo, y que daría pie a la proliferación de escuelas y facultades en los países latinoamericanos, que a partir de una mezcla de políticas económicas keynesianas y modelos administrativos norteamericanos, generaría un tipo específico de formación para entender el desarrollo como apuesta de orden técnico y profesional dentro de los establecimientos educativos y en pro del llamado bienestar social.

Una de las razones para justificar que fueran precisamente los países latinoamericanos los destinatarios prioritarios de esta reforma, fue el hecho de que en estos se encontraba la materia prima para volver a reactivar los motores de rentabilidad económica de los países del norte.

De acuerdo con Escobar (2007),

Las raíces históricas de esta nueva estrategia denominada "desarrollo" se encuentra en el reordenamiento político a nivel mundial que se produjo después de la Segunda Guerra Mundial y que dio paso a las denominadas naciones del "subdesarrollo" o "tercer mundo", las cuales fueron definidas en virtud de una nueva estrategia llamada "desarrollo" y de la cual se definirían las estructuras de poder globales (p. 429).

Así pues, "para todos los Estados subdesarrollados las políticas eran políticas de desarrollo, pues todas se concebían como políticas del catch up, o de cierre de la brecha que los separaba de los países desarrollados o industrializados" (Prats, 2010, p. 2) Las políticas de desarrollo, desde esta perspectiva, hicieron evidentes para la época una fuerte filiación utilitaria en la que los países denominados "en vías 
de desarrollo", a través de la adopción de los modelos de desarrollo impuestos, empezarían a generar indicadores económicos para alcanzar así, mejores niveles de bienestar comunitario y desarrollo.

"De ahí, que tanto el crecimiento per cápita como el PIB, pasarían a convertirse en el componente e indicador fundamental para medir las premisas del desarrollo" (Prats, 2010, p. 6). En consecuencia, el racionalismo técnico o instrumental partiría de la idea según la cual, ciencia, técnica y metodologías servirían de sustento para fortalecer y dar forma a las políticas de desarrollo a través de su inclusión como eje fundamental en el plano educativo.

Esta mirada utilitaria del bienestar pasaría a convertirse con el tiempo, en el casi único indicador para legitimar los niveles aceptables de desarrollo (Prats, 2010). Otro aspecto vigente es el hecho de vincular y en la mayoría de los casos referir la justicia social solo a la posibilidad de distribución de los bienes devenidos del crecimiento económico; elemento que, traducido a las condiciones de realidad, no representaría una condición necesaria para el desarrollo social y la producción cultural, y daría paso a la legitimación de un pensamiento abismal (De Sousa Santos, 2010, p. 16), entre pobres y ricos, entre aquellos que lideran sobre los patrones de poder (Quijano, 2000) y aquellos que sobreviven y sirven a dichos patrones.

Para el caso colombiano, las políticas desarrollistas - o lo que desde la historia de la invención de la idea de desarrollo podría bien llamarse una colonización de la realidad (Escobar, 2007) - tuvieron lugar tras la misión económica liderada por el Banco Internacional de Reconstrucción que visitaría a Colombia hacia 1947, con el fin de formular un programa general de desarrollo, como lo plantea Escobar (2007).

La misión contaba con catorce asesores internacionales en los siguientes campos: comercio exterior, transporte, industria, hidrocarburos y energía, vías carreteables y fluviales, servicios comunitarios, agricultura, salud y bienestar, banca y finanzas, economía, cuentas nacionales, vías férreas y refinerías petroleras (p. 50).

De esta misión liderada por el Banco Internacional de Reconstrucción surgió lo siguiente, en materia de acciones inmediatas:

Hemos interpretado nuestros términos de referencia como la necesidad de un programa integral e interior consistente. Las relaciones entre los diversos sectores de la economía colombiana son muy complejas, y ha sido necesario un análisis exhaustivo de las mismas para desarrollar un marco consistente. Esta, entonces, es la razón y justifica- ción para un programa global de desarrollo. Los esfuerzos pequeños y esporádicos solo pueden causar un pequeño efecto en el marco general. Solo mediante un ataque generalizado a través de toda la economía sobre la educación, la salud, la vivienda, la alimentación y la productividad puede romperse decisivamente el círculo vicioso de la pobreza, la ignorancia, la enfermedad y la baja productividad. Pero una vez que se haga el rompimiento, el proceso del desarrollo económico puede volverse autosostenido. (Escobar, 2007, p. 53)

En consecuencia, el dominio de un tipo específico de discurso apegado a lo económico, sería la directriz bajo la cual toda la lógica de cambio y transformación social se llevaría a cabo en Latinoamérica y en Colombia como parte integrante del conjunto de países subdesarrollados. La educación tomaría en este auge de reformas y re-definiciones, el lugar destinado antes a la fábrica, como la media base para la producción y desarrollo del país en busca del tan anhelado bienestar social (Martínez, 2010).

De ahí que la escuela como nueva fábrica se ocuparía de la transformación de la principal materia prima de la que gozan en abundancia los países así llamados tercermundistas. Su capital humano utilizable, el cual sería perfeccionado (Martínez, 2010) en aras de garantizar y optimizar los procesos de producción y construcción sistemática de las nuevas condiciones sociales necesarias para que se diera el desarrollo.

Así entendida, la capacitación del capital humano requería de cierta base administrativa tanto sencilla en su sentido operacional, como eficaz en sus resultados, por lo que, con la concepción de valor económico de la educación y su función en la formación de recursos humanos (Martínez, 2010) sobrevendría toda una construcción de sistemas integrados de información y de investigación de la aplicación de modelos a los nuevos escenarios educativos que permitiría un abordaje real de la relación entre educación y desarrollo y que, a su vez, brindaría una mirada diagnóstica a futuras decisiones en política educativa en el marco del desarrollo. En este sentido, y de acuerdo con las nuevas dinámicas para pensar el desarrollo, y en términos estrictamente económicos, una de las primeras variables que surgió fue la de producción.

Dicha variable, hacía referencia a patrones de organización de los factores de producción y rentabilidad económica, en el que se debía contemplar además de la producción directa, la relación residual que generaba la educación como aporte enorme en la producción, como lo llamaron los economistas de la educación, y la cual representaba y cumplía un papel 
fundamental en el incremento económico de la renta de los países. Pero esta idea de método residual no era nueva, ya que había servido con anterioridad para advertir cierto tipo de relación y correlación histórica entre el desarrollo científico y social del pueblo y su desarrollo económico. Así pues, y basado en suposiciones más que estudios históricos, surgía una nueva variable para el desarrollo en la que se vinculaba la educación como uno de los ejes del factor económico en pro del bienestar social.

Entre los temas centrales para el análisis económico de la educación, se propusieron tres ejes, que si bien no abonaron razones reales a la relación educación/economía, por lo menos, llevaron al surgimiento de una lógica transformista del espacio y de los modelos educativos para los países latinoamericanos. Estas variables o ejes de estudio serían:

- La posible influencia de la educación en el desarrollo económico.

- La influencia del desarrollo económico en el desarrollo educativo.

- La planificación educativa como estrategia para el desarrollo.

Con ello vendrían dos variables más, que servirían de apoyo y justificación al diseño y puesta en marcha del sistema de información a partir de dos vías: una empírica y otra teórica.

La primera consistiría en corroborar empíricamente la relación entre desarrollo y educación, mientras que la segunda se enfocaría en sustentar y justificar tal relación de manera teórica; de esto, se llegaría a la creación de dos métodos o sistemas de recolección de información. Uno llamado método de correlación en el que se establecería la relación directa entre el nivel de desarrollo educativo asociado con el nivel de desarrollo económico, y un segundo, llamado método residual, que se encargaría de valorar el crecimiento de la renta que tuviera el país, e intentar establecer una relación directa de dicho crecimiento, con la relación de factores cuantificables como trabajo, capital, etc.; ello ayudaría a determinar qué aspectos no estaban directamente asociados con el crecimiento de la renta y así se podría establecer otros factores residuales entre los que entraba la educación como gestora y productora de capital cognitivo utilizable.

Por esta razón, dentro de los métodos de estudio sobre producción, se incluyó el capital humano utilizable como una respuesta estratégica de los especialistas a los modelos tradicionales de medición y estudio de crecimiento y rentabilidad económica. Es decir, se empezó a medir el nivel educativo como un factor positivo o negativo de la economía, en el que el capital humano entraba como una variable para resolver la dificultad de los modelos económicos tradicionales para explicar el crecimiento económico en función exclusiva del comportamiento de los factores convencionales de capital y trabajo (Martínez, 2010), pasando así a incluir el factor educativo como uno de los efectos residuales del desarrollo cuya acción inmediata consistía en la capacitación de la mano de obra que daría la base para el despliegue del desarrollo.

En síntesis, se podría afirmar que el maridaje entre educación y desarrollo hizo énfasis de manera radical en un modelamiento de la realidad social en el que la escuela y todos sus elementos u objetos, por hacer uso del utillaje del desarrollo, pasaron a ser pensados como insumos y medios para alcanzar el crecimiento y desarrollo social esperado.

Así pues, y desde una mirada fundada en un racionalismo funcional se intentó modelar y destinar la función de la educación, dando forma al predominio de un tipo específico de pensamiento de base funcionalista y operativa, que debía ser implementado como parte de la estrategia educativa. No obstante, desde esta perspectiva lo que se puede leer en la historia, en relación al concepto de desarrollo, es una triple ambigüedad en la manera de concebirlo.

Esta ambigüedad tripartita podría ser enunciada como sigue:

- El modo disciplinar de la educación, en el que el maestro pasaba de ser un "administrador del currículo, a un facilitador de aprendizajes" (Martínez, 2010, p. 21).

- El modo institucional, en el que el Estado a través de políticas de corte económico trazaría las metas necesarias para pensar prospectivamente el devenir del modelo educativo para el país.

- El modo basado en las subjetividades de los actores de la educación, en el que las políticas desarrollistas empezarían aplicar todo un sistema de control a partir de la instauración de discursos formativos y su traducción en prácticas específicas.

Esta mirada variopinta del desarrollo, sin embargo, pasaría a formar la estructura material de los discursos institucionales y naturalizados por parte de los actores educativos a partir de modos y prácticas discursivas evidentes en la utilización de conceptos, enunciados y estrategias propias del desarrollo como indicadores y metas propias para pensar el desarrollo escolar. 
Así pues,

[...] dicho de una forma muy esquemática, lo que cambió y que no se hace evidente, fueron los procesos de mutación que afectan el tiempo escolar, la espacialidad de la educación, la subjetividad de la infancia, los procesos y los medios de enseñanza. (Martínez. 2010, p. 62).

Es decir, aquello que ha quedado inscrito en la memoria colectiva de los procesos de reestructuración social es una tendencia hacia lo económico vinculado a la educación, y a los sujetos que en esta se articulan y dan sentido desde una práctica en la que la educación, como institución es pensada como parte de un medio para la adquisición y mejora del capital económico de los países en vías de desarrollo. Es decir, como una educación que promete empleabilidad desde la necesidad como factor y promesa de formación.

De acuerdo con lo anterior, se podría pensar que "uno de los elementos distintivos del despliegue social de la estrategia del desarrollo, consistió en el inicio de un proceso al interior del cual la educación [...] conoció un conjunto de redefiniciones" (Martínez et al., 1994, p. 69). Así, la misma educación pasada por el filtro estratégico del desarrollo vendría a convertirse en parte de la estrategia, al ser pensada en términos de sus significados, objetivos y procedimientos como un bien producido para dar vía libre al desarrollo, y que llevaría a esta, a desbordar la tarea de constructora de nación, lo que pondría en entredicho la idea de bienestar social y cultural.

En consecuencia, el proceso de lo que podría llamarse una mundialización de la educación (Martínez et al., 2009), como dispositivo para el desarrollo, se vería evidenciada en la "expansión acelerada y vertiginosa de los sistemas educativos [...] en los procesos de modernización, en la aparición de nuevas teorías educativas, en el auge de la ciencia y la tecnología entre otros factores" (Martínez et al., 1994, p. 69) por lo que sería preciso advertir que:

El proceso de mundialización de la educación actuó, entonces, en una doble vía: a la vez que colocaba a la educación como instrumento indispensable para obtener mayores niveles de productividad (la educación como factor de producción) instauraba aquella en el horizonte de las necesidades de la población, como factor esencial de movilidad social (Martínez et al., 2009, p. 70).

En esta misma tónica, en los principios para el planeamiento del desarrollo como estrategia de evolución social ofrecido por la Unesco en 1962 se refiere a la educación como el lugar común tanto para la producción, como para el consumo:
En primer lugar, la educación es tanto un artículo de consumo como un factor de producción [...]; una segunda característica de la educación es que produce un margen muy elevado de ganancias indirectas [...]; un tercer rasgo de la educación es la gran diversidad de sus gastos y de su rendimiento según el nivel general de educación económica y social de un país. (Unesco, 1962, p. 8).

De ahí que la educación en la lógica del desarrollo solo sea un mecanismo de articulación de las dinámicas sociales economicistas y no como se le había supuesto, un mecanismo emancipatorio y generador de civilidad en un sentido ideológico de los fines supuestos a la educación. Es decir, como una educación que propende desde su acción educativa, por tejer relaciones de interacción comunitaria, social y humana tanto a nivel intra-subjetivo como intersubjetivo.

Así pues, el imperativo de ver en la educación un vehículo del desarrollo bajo una lógica de reproducción de patrones económicos de organización social, con el que se pretende dar respuesta a las necesidades sectoriales del país, podría dar lugar no solo a pensar la "educación en términos de productividad viable no solo para la sostenibilidad del país, sino también para construir una mejor relación entre desarrollo social y transformación cultural" (Granados, 2010, p. 58), a través de una tendencia tanto científica, tecnológica y desarrollista de la realidad social, como también, humana y cultural de esta. No se trata de negar de tajo el vínculo entre educación y desarrollo económico, pero tampoco se trata de reducir lo educativo solo a un rol económico, que si bien es importante y necesario, no contribuye al análisis contextual de las necesidades de orden social y cultural por las que atraviesa el país en la actualidad.

No obstante, de seguirse alimentando una concepción del desarrollo desde una perspectiva económica como única forma de expansión del capital social y cultural, se estaría apostando a la generación de una serie de consecuencias que lejos de ser inmediatas estarían dejando en el abandono a los entramados de relaciones humanas para privilegiar una reproducción en masa del capital cognitivo, que al decir de Granados (2010), terminaría “contribuyendo a la desintegración de la subjetividad y al sentido mismo de las relaciones personales" (p. 61) que antes se situaban en la escuela como lugar común para su formación y desarrollo. 


\section{Educación y desarrollo humano, otra perspectiva de un mismo modelo}

Pese al predominio de un modelo instituido de desarrollo desde una perspectiva económica, a partir de 1987 se pensó en una forma del desarrollo más cercano a lo humano. Dicha mirada sobre lo que en la historia se conocería como desarrollo humano, daría un giro a la manera como hasta la década de 1980 , se entendía en materia de producción y rentabilidad. El giro básicamente consistió, en dar una gran prelación a las capacidades humanas y desde allí engranar otra estrategia de producción, ya no basada en el producto, sino en el saber sobre la producción; en este giro la escuela tomaría de nuevo el lugar destinado a la estrategia de formación, acoplamiento y transformación de recursos humanos utilizables.

La nueva perspectiva de una educación para el desarrollo surgió, entonces, como una herramienta de transformación social y no solo de capitalización monetaria, radicalizando así, el uso histórico que se ha hecho del propio concepto de desarrollo en la cual se suele presentar como parte de una evolución humana, lo que significa, de acuerdo con Celorio y López (2007), que la definición de desarrollo debía acomodarse y evolucionar según el pensamiento y los valores dominantes de la sociedad y sus necesidades, y no a la relación directa con el capital financiero, quedando este segregado a valor agregado o derivado de la dinámica social, mas no como su única finalidad.

En este sentido, se estima que el desarrollo como concepto histórico está ligado a lo que "cada sociedad y cada época entiende o formula como desarrollo, y que responde, a las convicciones, expectativas y posibilidades que predominan en ellas" (Celorio y López, 2007, p. 76). De otro lado, el concepto de desarrollo alude a una noción de futuro, a lo que podrá ser, como parte de un proyecto mancomunado en el que la participación se relaciona con la posibilidad de llevar dicho concepto a la realidad; es decir, según lo descrito, el desarrollo implica lo deseable y lo legitimo, que debería configurar la aspiración y el ideario social de bienestar.

En consecuencia, al hablar de desarrollo se debe reflexionar sobre dos premisas fundamentales: la primera encaminada a resolver la cuestión, que podría plantearse como sigue: ¿Cuál sería el desarrollo deseable para una determinada sociedad, o, qué se debe establecer como prioridad fundamental para la sociedad? Y la segunda, indagar por cuál sería el desarrollo posible de acuerdo con las ambiciones o prioridades para cada sociedad y a sus condiciones reales; a decir, cuál de las prioridades anheladas serán realmente alcanzables.

Así pues, lo que cabría advertir en una educación para el desarrollo desde una perspectiva histórica, serían dos maneras de comprensión. Una que apunta a legitimar un modelo neoliberal en el que la lógica de la reproducción surge como el eje fundamental para el despliegue de toda la estrategia de crecimiento, y otra, basada en un modelo de potenciación de las capacidades humanas, como pilar del desarrollo y del propio crecimiento económico.

Los enfoques anteriores, en la historia han sido abordados desde varias intenciones y formas o modelos, que podrían ser enunciados como siguen:

- Enfoque caritativo asistencial.

- Nacimiento de la educación para el desarrollo bajo el enfoque desarrollista.

- Educación para el desarrollo, crítica y solidaria.

- Educación para el desarrollo humano y sostenible.

- La educación para el desarrollo y la ciudadanía global.

Estos enfoques, al parecer de Celorio y López (2007), brindan un acercamiento general a la relación educación y desarrollo, y pueden dar una idea de cómo cada modelo de una educación para el desarrollo puede ser leído en términos de una creciente necesidad de ajuste y cambio social sucesivas y ligadas a un contexto determinado.

En este sentido, y partiendo de la premisa sobre la que descansa el desarrollo como concepto histórico y como parte del reflejo o parte de una promesa social, se podría afirmar que se fragua el nicho para que surja el concepto de desarrollo humano (DH). Este, históricamente, toma forma institucional a principios de los años 1990, impulsado inicialmente por el Programa de las Naciones Unidades para el Desarrollo (PNUD), y previsto ya desde la década de 1980, por el Centro de Estudio y Promoción de Asuntos Urbanos (Cepaur), en el cual, se establecía la necesidad de vincular a la economía, las necesidades de los individuos. De ahí que hablar de desarrollo supondría, de acuerdo con el Cepaur, un cambio radical a las concepciones mantenidas hasta el momento, y que deberían fijar el nuevo rumbo del desarrollo.

Por consiguiente, desde el PNUD como mirada instituida (Bourdieu y Passeron, 2009), se estimó, que el "desarrollo sería el fundamento del proceso por el que se expandirían o ampliarían las capacida- 
des de las personas para que estas pudieran elegir su modo de vida" (Celorio y López, 2007, p. 80). Esta mirada sobre el desarrollo expuesta por el PNUD recalca la necesidad de pensar el desarrollo más allá de la expansión del capital, y de incluir dentro de la variable del desarrollo el activo humano.

Así pues, y de acuerdo con el Grupo de Educación de la Coordinadora de ONG para el Desarrollo (2005)

La cultura de la solidaridad es un elemento esencial en cualquier forma de desarrollo, entendido el desarrollo como una forma de garantizar a todos y todas la igualdad de condiciones, tanto materiales como espirituales; condiciones a su vez necesarias, para una existencia digna como ser humano, así entendido el desarrollo, es una forma constructivista de referirse a la lucha contra la pobreza (p.14)

Así, la educación para el desarrollo (ED) surge como una promesa no solo de potenciación de cada individuo, sino además, de la posibilidad de un impacto significativo en el ámbito comunitario, de ahí, que se hable de una educación para el desarrollo regional o local, pues se entiende que la ED debe influir sobre todo el contexto inmediato en el que habitan los individuos, promoviendo así, un despliegue social en el que la escuela representa un factor fundamental como eje para alcanzar el desarrollo deseado.

No obstante, y según Celorio y López (2007), “la piedra angular para que el desarrollo humano se convierta en una propuesta alternativa se encuentra en la definición que se haga del concepto de bienestar" (p. 81). Esto supone unas preguntas adicionales que se deben enunciar, según los términos, como: ¿Cómo entender el bienestar posible y deseable de las personas? ¿Cuál sería el margen intermedio para no malograr una premisa sobre el desarrollo que intente dar una mirada al bienestar?

Orduna y Naval (2011) plantean que se debe indagar por el significado que se tenga sobre el desarrollo y de cómo este se determina en relación a las necesidades sociales inmediatas. De ser posible esto, se permitiría una distinción necesaria sobre el desarrollo, pues así se sabría a qué se está aludiendo, si a "un desarrollo para la supervivencia y expansión económica, o a un desarrollo para la promoción y el crecimiento humano" (Orduna y Naval, 2011, p. 7).

Para efectos del presente estudio, la definición que se adopta sobre el desarrollo humano corresponde a la que designa la Organización de las Naciones Unidas (ONU), en la cual, y según Orduna y Naval (2011), se entiende como:
Un modelo o paradigma de desarrollo que coloca a los individuos (y no a su mero progreso económico) en el centro de sus preocupaciones, es un proceso de ampliación de las opciones de que dispone el ser humano, de todos los individuos y no solo de una parte de la comunidad, con el propósito de crear un ámbito en el que esas personas disfruten de una vida larga y saludable, puedan adquirir conocimiento y puedan tener acceso a los recursos necesarios para disfrutar de un nivel de vida decoroso (pp. 8-9).

El desarrollo, así entendido, tiene dos facetas: por un lado, el fenómeno de la capacidad humana, como mejor salud y mayores conocimiento teóricos y prácticos -educativos-, y por otro lado, el aprovechamiento de la capacidad adquirida por las personas, que llevaría a lo que Malassis (1975, citado por Orduna y Naval, 2011) denomina innovación. El desarrollo, desde esta perspectiva, supondría una organización de las personas según su comunidad para alcanzar su desarrollo, a través de cambios sociales y económicos que han de ser innovadores.

En consecuencia a estas dos perspectivas, se supondría que un posible desarrollo humano se encuentra sustentado en la premisa de la producción, como base para que las personas puedan incluirse en las dinámicas sociales de generación de ingresos y empleo, y de ahí, derivar la equidad, asumida esta como el acceso distributivo a las oportunidades de orden económico, es decir, que vayan en pro de la neutralización de la pobreza relativa, pues se entiende equidad como la posibilidad de acceder y adquirir bienes.

Por tanto, pese a que se hable de un desarrollo humano en el que se incluye lo educativo como una práctica que propende por potenciar lo humano a través de la formación, el hecho de hacer depender el concepto de bienestar de la posibilidad del despliegue económico sesga la mirada misma sobre cómo se deberá entender el bienestar como concepto dentro de las dinámicas del desarrollo. Así, como ya se describió, tanto la concepción que se tenga del bienestar, como la que predomine sobre el desarrollo, serán los indicadores clave para establecer cómo y en qué sentido se está pensando el despliegue de una política sobre el desarrollo, además de permitir y advertir si dicha política apunta o no a justificar en lo humano la necesidad de su proyección.

Entonces, se puede corroborar que pese a que existan varias formas de comprender el desarrollo, la concepción que se tiene de este, desde los organismos estatales e internacionales, siempre termina redundando en la posibilidad de hacer un despliegue 
del capital financiero de los países, como condición y espíritu mismo de la política económica tanto para su desarrollo, como para el de su comunidad.

Sin embargo, lo que se estima dentro de las comprensiones, significaciones y nociones que se tienen sobre desarrollo, como concepto histórico, y a su inclusión en el plano educativo, se podría considerar que la educación - por su naturaleza y según la concepción transformadora y renovadora de los procesos sociales con la que se le ha concebido-, impactaría, de forma directa, positiva y significativa mediante un juego de doble vía; es decir, en la medida en que potencia a los individuos, serviría para potenciar el desarrollo social de las comunidades a través de cuatro principios derivados del desarrollo de acuerdo con la concepción designada desde la onU.

En este sentido, cabría hablar de una educación para el desarrollo, contemplada desde una especie de acción educativa temprana que posibilitaría al individuo un nivel intereducativo tanto en una vía subjetiva como intersubjetiva, del concepto de formación, de ahí el carácter de aprendizaje social, el cual debería estar en vigilancia de un adulto, como lo propone Unicef (1999), que permita la integración entre la acción educativa específica y el aprendizaje social esperado para el desarrollo de la comunidad. De ahí, que el adulto, en este caso el docente, sea visto como un tipo específico de educador social.

Asumido el desarrollo como un proceso de aprendizaje social, en el cual el docente actúa como algo más que un simple administrador y gestor de información, podría contribuir a una implicación de la comunidad en la gestión de sus propios fines y necesidades, al tener la capacidad de identificar y traducir sus necesidades y visiones sobre su propio desarrollo a partir de proyectos acordes a su contexto histórico, económico, social y ecológico (Sachs, 1978, citado por Orduna y Naval, 2011).

En esta propuesta, en la que no se deja de lado el espíritu financiero del desarrollo, y se incluyen las variables de comunidad y bienestar social como formas arquetípicas de lo humano, se podría pensar que parte de la dinámica del desarrollo está basado en un tipo específico de aprendizaje social, en el cual, la escuela cumple un papel fundamental, pues de acuerdo con Orduna y Naval (2011), el desarrollo, "como aprendizaje social, demanda la participación responsable y activa de la población, apoyándose, en consecuencia, en la educación como medio para capacitar a la población misma" (p. 14).
En ese sentido,

Educar para el futuro es educar para ser protagonistas del cambio. El hombre debe estar preparado para desarrollar su vida y sus posibilidades en un momento histórico totalmente cambiante e incierto pero real. Es preciso, entonces, ofrecer a los miembros de la sociedad una educación centrada tanto en el sujeto que aprende, adaptada a su situación, a sus necesidades, y a sus aspiraciones, como a las de su comunidad. (Escotet, 1986, citado por Orduna y Naval, 2011, p. 14).

Así una educación para el desarrollo que pueda incluir en sus agendas la promoción de cada individuo a partir de un proceso educativo sistemático y de impacto social en pro de los miembros de la comunidad, cabría como una educación de carácter social, y no solo como una premisa o mecanismo de despliegue económico para dar vía libre al desarrollo financiero y productivo de un determinado país.

De ahí, pues, que la educación, en especial aquella que se funda en una concepción intereducativa, pueda lograr un proceso en doble vía, es decir, una educación que potencie las estrategias de autoeducación, pero que no olvide el carácter hetereoeducador, es decir, "entre la acción inmanente del educando en el caso del desarrollo local-que se compromete a educarse a sí mismo, y la acción transeúnte del educador, la ayuda que este presta a cada individuo, sea quien sea el educador" (pp. 14-15).

Bajo esta mirada humanista, el desarrollo podría considerarse tal y como lo conciben Gonzales y Simancas (1992, citados por Orduna y Naval, 2011).

La educación para el desarrollo debería promocionar a todo el hombre. A todos los hombres. Un proceso esencialmente dinámico de comunicación entre dos o más seres que pretende el perfeccionamiento del individuo como persona y busca la inserción activa y consciente del ser personal en la mejora del mundo social (pp. 36-37).

Del mismo modo, Herreros (1990, citado por Orduna y Naval, 2011) plantea que la educación para el desarrollo, entendida como promoción y crecimiento, "debe contribuir, en primer lugar a que cada persona sea mejor valorada por lo que es y lo que es capaz de hacer. En segundo lugar, debe favorecer el éxito de las iniciativas, y la viabilidad de las empresas" (p. 15).

Lo anterior implica un ciclo en el cual se legitima un proceso de aprendizaje basado o soportado en la premisa de mejora de las condiciones de vida en comunidad, que al decir de Orduna y Naval (2011), se da a través del "fomento de actitudes, y despliegues 
de destrezas, individuales y colectivas, necesarias para detectar problemas, diagnosticarlos, estudiarlos, plantear posibles soluciones y actuar en consecuencia" (p. 17). De ahí que lo que se deba pensar como una educación para el desarrollo en la perspectiva que se ha descrito en la segunda parte del presente estudio deba considerarse como un concepto cuyo alcance es mucho más complejo y diverso, en tanto se debe comprender como parte de una acción dinámica del propio proceso de construcción social del conocimiento.

De ahí pues, que de ser posible una concepción sobre lo educativo que albergue las variables de acción educativa, intereducación, aprendizaje social y educador social, como la base para la formación y la transformación social del individuo y de la comunidad, se estaría apostando por un nivel del desarrollo a una escala más compleja que la mantenida hasta ahora desde la mirada de la expansión del capital. Entonces, de sucederse una educación para el desarrollo desde lo descrito, se estaría potenciando la participación de cada individuo de la comunidad como un agente de desarrollo cuyo eje fundamental de acción estaría basado en estimular y participar activamente en el tejido social y hacia la construcción de nuevos y cada vez mejores valores sociales basados en los principios de cooperación y solidaridad.

Dichas variables son las que forman el grupo de categorías a ser indagadas en la noción que sobre lo educativo prevalece en los actores educativos del departamento de Caldas, y que se espera pueda brindar una radiografía de un estado real del impacto que la política desarrollista ha tenido tanto en las dinámicas de la expansión de la estrategia económica, sino también desde la perspectiva humana.

\section{Aproximación al método del estudio}

Para el presente estudio, la perspectiva metodológica se enmarcó en un enfoque cuantitativo cuyo alcance fue de carácter descriptivo/correlacional los cuales según Hernández et al. (2010), "busca especificar propiedades, características y rasgos importantes de cualquier fenómeno, describiendo tendencias de un grupo o población" (p. 80). De igual manera, los mismos autores definen el carácter correlacional de la investigación, como aquella que "asocia variables mediante un patrón predecible para un grupo o población" (p. 81). Con este diseño, se pudo establecer la descripción de cuatro categorías asociadas con una educación para el desarrollo, y rastrear su uso o no, en la concepción presente en el discurso que los estudiantes, maestros y directivos tienen sobre educación en el departamento de Caldas.

\section{Descripción del análisis y manejo datos del estudio}

Los datos obtenidos en el estudio fueron analizados en tres momentos: hallazgo del índice de frecuencia, coeficiente de Pearson y coeficiente de determinación. Así, el grado de correlación de acuerdo con el coeficiente hallado hace posible interpretar que a mayor incidencia o recurrencia de una variable, esta afectará o influirá sobre el componente y componente correlacionado. El componente DE está constituido por dos subcomponentes o categorías: educador social (ES) y aprendizaje social (AS). El componente DH está constituido por dos componentes: acción educativa (AD) e inter-educación (IE).

\section{Participantes}

La población representada en este estudio corresponde al conjunto de estudiantes del departamento de Caldas, sector oficial, de la zona urbana y matriculados en grado undécimo, de los municipios de La Dorada, Manzanares, Manizales, Neira, Riosucio y Viterbo. Para estimar la unidad de análisis, fue necesario calcular los datos a partir del total de matrículas reportadas para el año 2013, y sobre dicho número se estimó una reducción gradual de matrículas para 2014, a razón de un comportamiento de tendencia a la baja de $-1,2 \%$, de lo que resultó un estimativo de 6086 estudiantes matrícula total. Sobre dicho estimativo de matrículas para el departamento de Caldas se realizaron los correspondientes estadísticos para conocer la unidad de análisis así: total de la población (n) 6086 nivel de confianza $95 \%$; tamaño de aproximación $50 \%$; error muestras estimado $5 \%$. De una población $(n)$ se estima la unidad de análisis en 387p. Como muestra significativa de la población total. Para la captación de datos a docentes y directivos docentes, la unidad de análisis se estimó en relación a la posibilidad de atención y respuesta voluntaria, de tal forma que se logró aplicar el instrumento a:

\section{Estrategia analítica}

Para estimar la relación entre las categorías alusivas al desarrollo y su implementación en el uso/ frecuencia que los sujetos hacen cuando hablan y nominan lo educativo, se partió del diseño de una encuesta estructurada compuesta por cinco ítems, 
cada uno constituido por cuatro respuestas asociadas de manera implícita a los subcomponentes antes citados. Cada ítem o pregunta indagaba de manera directa por tendencias, concepciones y gustos que tanto estudiantes, como docentes y directivos tienen sobre lo educativo y su práctica. Una vez aplicado el instrumento a la población muestra descrita en la tabla 3 , se procedió a realizar la tabulación y análisis de los datos a partir del software: IBM SPSS v.22, en el que se hizo el manejo de datos del estudio. Para ello se definió un índice debido a la frecuencia de respuestas por asignación de categorías que se denominó índice de frecuencias, que tiene una definición en común a la frecuencia relativa usada en la estadística ${ }^{\text {descriptiva }} I_{f}=\frac{n_{c}}{N}$ (Solanas et al. 2005)

Donde $n_{c}$ el número de personas con afinidad hacia la categoría y $N$ el número de personas por segmento. Posteriormente, se describieron correlaciones entre las categorías de estudio, para visualizar posibles relaciones.

\section{Análisis y resultados}

Tabla 4. Índices de frecuencias $\left(I_{f}\right)$ por municipio Neira/Riosucio.

\begin{tabular}{|l|c|c|c|c|c|c|}
\hline \multirow{2}{*}{ MUNICIPIO } & \multicolumn{3}{|c|}{ NEIRA } & \multicolumn{3}{c|}{ RIOSUCIO } \\
\cline { 2 - 8 } & ESTUDIANTES & DOCENTES & DIR.DOCENTES & ESTUDIANTES & DOCENTES & DIR.DOCENTES \\
\hline SUBCOMPONENTE & & & & & & \\
\hline INTEREDUCACIÓN & 0,17 & 0 & 0,23 & 0,24 & 0,3 & 0,15 \\
\hline ACCIÓN EDUCATIVA & 0,41 & 0,6 & 0,33 & 0,25 & 0,27 & 0,375 \\
\hline EDUCADOR SOCIAL & 0,27 & 0,4 & 0,28 & 0,36 & 0,3 & 0,34 \\
\hline APRENDIZAJE SOCIAL & 0,13 & 0 & 0,14 & 0,16 & 0,11 & 0,125 \\
\hline
\end{tabular}

(Fuente: elaboración propia)

Tabla 5. Índices de frecuencias $\left(I_{f}\right)$ por municipio La Dorada/Manizales (Fuente: elaboración propia)

\begin{tabular}{|l|c|c|c|c|c|c|}
\hline \multirow{2}{*}{ MUNICIPIO } & \multicolumn{3}{|c|}{ LA DORADA } & \multicolumn{3}{c|}{ MANIZALES } \\
\cline { 2 - 7 } & ESTUDIANTES & DOCENTES & DIR.DOCENTES & ESTUDIANTES & DOCENTES & DIR.DOCENTES \\
\hline SUBCOMPONENTE & & & & & & 0,2 \\
\hline INTEREDUCACIÓN & 0,26 & 0,25 & 0,18 & 0,15 & 0,23 \\
\hline ACCIÓN EDUCATIVA & 0,28 & 0,27 & 0,4 & 0,29 & 0,38 & 0,47 \\
\hline EDUCADOR SOCIAL & 0,38 & 0,33 & 0,18 & 0,31 & 0,26 & 0,19 \\
\hline APRENDIZAJE SOCIAL & 0,14 & 0,13 & 0,21 & 0,23 & 0,15 & 0,09 \\
\hline
\end{tabular}

(Fuente: elaboración propia)

Tabla 6. Índices de frecuencias $\left(I_{f}\right)$ por municipio Manzanares/Viterbo (Fuente:elaboración propia)

\begin{tabular}{|l|c|c|c|c|c|c|}
\hline \multirow{2}{*}{ MUNICIPIO } & \multicolumn{3}{c|}{ MANZANARES } & \multicolumn{3}{c|}{ VITERBO } \\
\cline { 2 - 8 } & ESTUDIANTES & DOCENTES & DIR.DOCENTES & ESTUDIANTES & DOCENTES & DIR.DOCENTES \\
\hline SUBCOMPONENTE & & & & & & 0,3 \\
\hline INTEREDUCACIÓN & 0,23 & 0,16 & 0,23 & 0,14 & 0,28 \\
\hline ACCIÓN EDUCATIVA & 0,35 & 0,45 & 0,35 & 0,38 & 0,45 & 0,41 \\
\hline EDUCADOR SOCIAL & 0,26 & 0,08 & 0,17 & 0,25 & 0,15 & 0,14 \\
\hline APRENDIZAJE SOCIAL & 0,14 & 0,16 & 0,23 & 0,21 & 0,1 & 0,15 \\
\hline
\end{tabular}

(Fuente: elaboración propia) 
Tabla 7. Índices generales municipios. Mediana/Desviación estándar.

\begin{tabular}{|l|c|c|}
\hline \multicolumn{1}{|c|}{ SUBCOMPONENTE } & MEDIANA & DESV. STA. \\
\hline INTEREDUCACIÓN & 0,22 & 0,05 \\
\hline ACCIÓN EDUCATIVA & 0,37 & 0,09 \\
\hline EDUCADOR SOCIAL & 0,26 & 0,09 \\
\hline $\begin{array}{l}\text { APRENDIZAJE } \\
\text { SOCIAL }\end{array}$ & 0,15 & 0,04 \\
\hline
\end{tabular}

(Fuente: elaboración propia)

Según lo que se expresa en relación al índice de frecuencia, se hace evidente una tendencia marcada del uso de la categoría acción educativa, la cual de acuerdo con el rango de respuestas de los estudiantes y a su concepción sobre lo educativo, resultó prevalente. Así se muestra cómo, de acuerdo con Puelles (1982, citado por Orduna y Naval, 2001), la concepción mantenida por los estudiantes del departamento de Caldas, se entiende como la acción que permite al ser humano avanzar hacia el estado de progreso que todo hombre encierra como potencia. Es decir, aquello que cada quien, por el hecho de ser educado, debería alcanzar en términos del conjunto de conocimientos, aptitudes, competencias y comportamientos en pro de su bienestar.

Entonces, se hace evidente que, a partir de la mediana extraída del consolidado del índice de frecuencia de respuesta en el total de los municipios, se obtiene que se mantiene una tendencia hacia un uso naturalizado de lo educativo desde una perspectiva del desarrollo humano, salvo por el municipio de La Dorada que presentó un índice de frecuencia en el uso nominativo que se le da a lo educativo inclinado a una concepción desde su utilidad económica.

No obstante, la mediana ${ }^{1}$ que es el valor relevante con el que se entrará a realizar la covarianza de los análisis posteriores, muestra una marcada tendencia hacia la categoría $\mathrm{AE}$, cuyo componente base es el desarrollo humano. Esta inclinación hacia la categoría AE se corrobora en la tabla 6, de índice de respuesta por total de estudiantes.

Tabla 8. Índices de respuesta por estudiantes. Mediana/Desviación estándar

\begin{tabular}{|l|c|c|c|c|c|c|c|c|}
\hline & NEIRA & RIOSUCIO & LA DORADA & MANIZALES & MANZANARES & VITERBO & MEDIANA & $\begin{array}{c}\text { DESV. } \\
\text { STAN. }\end{array}$ \\
\hline INTEREDUCACIÓN & 0,17 & 0,24 & 0,26 & 0,15 & 0,23 & 0,14 & 0,2 & 0,0512 \\
\hline $\begin{array}{l}\text { ACCIÓN } \\
\text { EDUCATIVA }\end{array}$ & 0,41 & 0,25 & 0,28 & 0,29 & 0,35 & 0,38 & 0,32 & 0,0628 \\
\hline $\begin{array}{l}\text { EDUCADOR } \\
\text { SOCIAL }\end{array}$ & 0,27 & 0,36 & 0,38 & 0,31 & 0,26 & 0,25 & 0,29 & 0,0547 \\
\hline $\begin{array}{l}\text { APRENDIZAJE } \\
\text { SOCIAL }\end{array}$ & 0,13 & 0,16 & 0,14 & 0,23 & 0,14 & 0,21 & 0,15 & 0,0417 \\
\hline
\end{tabular}

(Fuente: elaboración propia)

Teniendo en cuenta los resultados obtenidos en la tabla 8, la mediana obtenida de los índices de frecuencias más alta corresponde al subcomponente de AE con 0,32 con una desviación estándar baja de 0,06 , medida que evidencia la cercanía en la valoración al comparar el resultado de los índices de frecuencia obtenidos por municipios (tabla 7).

Por consiguiente, la consecución del subcomponente AE para el análisis del índice de frecuencia por grupos de estudiantes (tabla 8) resulta significativo, pues indica la prevalencia y naturalización en el discurso de una concepción sobre lo educativo desde la perspectiva del desarrollo humano, aspecto que podría llevar a pensar que el valor adjudicado a lo educativo va más allá de su uso instrumental y económico como cabría suponer después de la revisión de las políticas de administración educativa por la que ha atravesado el sistema educativo colombiano.
En ese sentido, y de acuerdo con Herreros (1990, citado por Orduna y Naval, 2001), la frecuencia o uso en el lenguaje de este subcomponente es un indicio de la búsqueda por mejorar las condiciones de vida tanto del individuo como de la comunidad a través del despliegue de conocimientos, fomento de actitudes y de destrezas, tanto individuales como colectivas, con miras a la resolución y prevención de problemas, lo que enmarca una mirada sobre lo educativo como base para el desarrollo social y comunitario.

Del mismo modo, el resultado es significativo en la medida que establece, teniendo en cuenta el índice de frecuencia, una correspondencia con la subcategoría educador social (ES) con un índice de 0,29.

1 Se tomó la mediana, ya que la naturaleza de las muestras en el análisis de índice de frecuencia mostró un nivel muy alto de heterogeneidad. 
De acuerdo con Cervera (1974, citado por Orduna y Naval, 2001) esta categoría demanda la participación responsable y activa de la población, apoyándose, en la educación como medio para generar cambios significativos en la población.
En la misma medida, y en relación al soporte teórico que funda este componente, este se define, en términos de la estimulación y participación social hacia el favorecimiento de la articulación del tejido asociativo, potenciados en valores de cooperación y solidaridad (Orduna y Naval, 2001, p. 16).

Tabla 9. Índices de respuesta por docentes. Mediana/Desviación estándar

\begin{tabular}{|l|c|c|c|c|c|c|c|c|}
\hline & NEIRA & RIOSUCIO & LA DORADA & MANIZALES & MANZANARES & VITERBO & MEDIANA & $\begin{array}{c}\text { DESV. } \\
\text { STAN. }\end{array}$ \\
\hline INTEREDUCACIÓN & 0,1 & 0,3 & 0,25 & 0,2 & 0,16 & 0,3 & 0,225 & 0,0801 \\
\hline $\begin{array}{l}\text { ACCIÓN } \\
\text { EDUCATIVA }\end{array}$ & 0,6 & 0,27 & 0,27 & 0,38 & 0,45 & 0,45 & 0,415 & 0,1258 \\
\hline $\begin{array}{l}\text { EDUCADOR } \\
\text { SOCIAL }\end{array}$ & 0,4 & 0,3 & 0,33 & 0,26 & 0,08 & 0,15 & 0,28 & 0,1186 \\
\hline $\begin{array}{l}\text { APRENDI- } \\
\text { ZAJE SOCIAL }\end{array}$ & 0 & 0,11 & 0,13 & 0,15 & 0,16 & 0,1 & 0,12 & 0,0578 \\
\hline
\end{tabular}

(Fuente: elaboración propia)

El análisis por índice de frecuencia para el caso de los docentes (tabla 9) muestra una correspondencia en los subcomponentes prevalentes en los datos obtenidos en las tablas 7 y 8 respectivamente. De donde se corrobora que la mediana más alta se inclina hacia el subcomponente $\mathrm{AE}$ con 0,415 y ES con 0,28 . La relación de estos dos subcomponentes sigue confirmando que el uso nominativo que se le da a lo educativo y al uso discursivo que se ha construido sobre lo educativo, se encuentra emparentado con una tendencia de potenciador no solo del individuo, sino de la comunidad en general, aspectos asociados al desarrollo humano.

El uso nominativo sobre lo educativo como una especie de agente del desarrollo humano, podría explicarse desde la naturaleza de los propios docentes, pues dentro de su ejercicio laboral está la promoción de los contenidos curriculares en pro del desarrollo social, según las descripciones que de ambos subcomponentes se realizaron para las tablas 7 y 8 .

Tabla 10. Índices de respuesta por Dir. Docentes. Mediana/Desviación estándar

\begin{tabular}{|l|c|c|c|c|c|c|c|c|}
\hline & NEIRA & RIOSUCIO & LA DORADA & MANIZALES & MANZANARES & VITERBO & MEDIANA & DESV. STAN. \\
\hline INTEREDUCACIÓN & 0,23 & 0,15 & 0,18 & 0,23 & 0,23 & 0,28 & 0,23 & 0,0455 \\
\hline ACCIÓN EDUCATIVA & 0,33 & 0,375 & 0,4 & 0,47 & 0,35 & 0,41 & 0,3875 & 0,0496 \\
\hline EDUCADOR SOCIAL & 0,28 & 0,34 & 0,18 & 0,19 & 0,17 & 0,14 & 0,185 & 0,0766 \\
\hline APRENDIZAJE SOCIAL & 0,14 & 0,125 & 0,21 & 0,09 & 0,23 & 0,15 & 0,145 & 0,0529 \\
\hline
\end{tabular}

(Fuente: elaboración propia)

De forma similar a los docentes, y en concordancia con los datos obtenidos en las tablas anteriores, los directivos muestran un uso nominativo sobre lo educativo con preferencia al uso/frecuencia de la categoría AE. A partir del análisis que arrojó el índice de frecuencia, el mayor valor se vio inclinado para la categoría AE, en relación a los demás con 0,3875 siendo la mayor en el conjunto de los datos, seguida de IE con 0,23.

Así pues, la concordancia de la categoría AC y su correlación positiva con la categoría IE evidencia la preocupación que desde los directivos de las instituciones educativas se da, por hacer uso de las premisas de la política pública educativa, toda vez, que la intereducación se define como un proceso en doble vía; es decir, como posibilidad tanto de autoeducación, como de heteroeducación. Esta relación, según Orduna y Naval (2001),

[...] consiste en la relación íntima que se da entre la acción inmanente del educando en el caso del desarrollo local, las personas que integran grupos que participan en la vida comunitaria - que se comprometen a educarse a sí mismos-y la acción transeúnte del educador, que se manifiesta en la 
ayuda que este presta a cada individuo, sea quien sea el educador (generalmente, el educador social), pero también otros sujetos, los grupos o, incluso, la comunidad (p. 14).

De igual manera, la intereducación como propuesta educativa, se centra en las nociones de intervención educativa y principio de crecimiento personal, abonados desde la acción educativa para dar forma a una propuesta educativa en la que se propende por la formación tanto humana como intelectual de la persona humana.

\section{Análisis de datos por correlación}

Para realizar el análisis de los datos por medio de la correlación entre los componentes, se generaron, a manera de ejemplo, algunos modelos gráficos con los que se pretende aclarar más la relación y correlación que se llevará a cabo.
Así pues, la gráfica 1 busca ilustrar la orientación que se propuso a partir de los resultados de los índices de frecuencia mostrados en las tablas $4,5,6,7$, 8 y 9. En ese sentido, para el componente desarrollo humano (DH), según la gráfica 1 , se correlacionará con el componente desarrollo económico (DE) a través de sus subcomponentes o categorías asociadas, de tal forma que la categoría acción educativa (AE) se correlacionará con la categoría educador social (ES). De igual manera se hará con las categorías sucesivas

A partir de lo que se muestra en modelo de correlación, en la tabla 11 se analizan los valores obtenidos en el índice de frecuencia, en concordancia a la correlación de cada una de las categorías con su componente opuesto a través del coeficiente de correlación lineal. Posteriormente, dentro de la misma tabla, se estudia el coeficiente de determinación $R^{2}$, cuya finalidad es fijar la proximidad (fortaleza de correlación) en la correlación de los datos de las tablas 2 a 7 , según la fortaleza de cada correlación inicial, y a su valor de coeficiente de correlación lineal.

Tabla 11. Coeficiente de correlación lineal y coeficiente de determinación $R^{2}$ de la correlación, según los valores obtenidos para cada subcategoría en el índice de frecuencia. Submuestras: Dir. Docente, docentes y estudiantes.

\begin{tabular}{|l|l|c|c|}
\hline FUN. CORRELACIÓN & & Coef.Corl & R2 \\
\hline DH/DE & ACCIÓN EDUCATIVA - EDUCADOR SOCIAL & $-0,3060249$ & 0,09365127 \\
\hline DH/DH & ACCIÓN EDUCATIVA - INTER/EDUCACIÓN & $-0,5708813$ & 0,32590551 \\
\hline DE/DH & APRENDIZAJE SOCIAL - INTER/EDUCACION & 0,191845 & 0,03680449 \\
\hline DE/DE & EDUCADOR SOCIAL - APRENDIZAJE SOCIAL & $-0,3250255$ & 0,10564156 \\
\hline
\end{tabular}

(Fuente: elaboración propia)

En la tabla 11, el coeficiente de determinación $R^{2}$ evidencia que la correlación que mejor representación lineal posee obedece a los subcomponentes de AE e IE.

Según el componente al que pertenece cada subcomponente, y a la proximidad hallada por el coeficiente de determinación $R^{2}$, es posible advertir - sustentados en la teoría-, que teniendo en cuenta las personas consultadas y las diversas acciones lideradas por la escuela en términos del desarrollo como acción comunitaria, esta relación se encuentra positivamente correlacionada con las formulaciones que se dan en el espacio educativo acerca del carácter que se presupone a la función educativa, así como al desarrollo próximo de la comunidad a través de un proceso exitoso de escolarización.

No obstante, la misma tabla 11 muestra un coeficiente de correlación lineal más alto para la relación entre los subcomponentes AS e IE, lo que implica según los datos obtenidos en el índice de frecuencia, y al soporte manejado desde la perspectiva teórica, un giro hasta ahora poco evidente, en el que se muestra en el uso nominativo de los subcomponentes alusivos al discurso educativo un sesgo interesante, pues pese a que existe casi consenso en la idea sobre lo educativo como un motor o base para el desarrollo humano y social de la comunidad, surge una condición para que esto se dé, de acuerdo con lo arrojado por el coeficiente de correlación lineal.

A partir de los datos obtenidos en el coeficiente de correlación lineal, la posibilidad para que sea posible una concepción sobre lo educativo naturalizada en el discurso, como base para el desarrollo humano, se encuentra ligado a la posibilidad que lo educativo tenga un impacto significativo en el desarrollo económico de la comunidad y en el uso de plataforma adquisitiva de los individuos escolarizados.

Así pues, con los datos del coeficiente de correlación lineal, se tiene que el desarrollo económico presenta un estadístico de correlación de 0,191844955, 
sobre el desarrollo humano con un estadístico de correlación de 0,036804487 , correlación que llevaría a plantear, que con los datos obtenidos en las tablas anteriores, el rasgo de posibilidad para que se dé el desarrollo humano como una manifestación no solo discursiva, sino pragmática, se encuentra supeditada al impacto económico que la educación como mecanismo y agente del desarrollo pueda lograr.

Se puede, entonces, corroborar cómo el discurso de las políticas públicas acerca de la necesidad de hacer de la educación un vehículo de empleabilidad a través de la formación técnica y tecnológica como se plantea en la propuesta de articulación de la educación no formal (técnica y tecnológica) de entidades como el Servicio Nacional de Aprendizaje (SENA), institutos técnicos, etc., con la educación formal y el sistema nacional de formación para el trabajo. Del mismo modo, esto entra en armonía con lo consagrado en el capítulo I del Decreto 4904 de 2009, en los numerales 1.2 y 1.3 e incisos.

\subsection{Educación para el trabajo y el desarrollo}

humano. La educación para el trabajo y el desarrollo humano forma parte del servicio público educativo y responde a los fines de la educación consagrados en el artículo 5 de la Ley 115 de 1994. Se ofrece con el objeto de complementar, actualizar, suplir conocimientos y formar, en aspectos académicos o laborales y conduce a la obtención de certificados de aptitud ocupacional.

Comprende la formación permanente, personal, social y cultural, que se fundamenta en una concepción integral de la persona, que una institución organiza en un proyecto educativo institucional y que estructura en currículos flexibles sin sujeción al sistema de niveles y grados propios de la educación formal.

1.3. Objetivos. Son objetivos de la educación para el trabajo y el desarrollo humano:

1.3.1. Promover la formación en la práctica del trabajo mediante el desarrollo de conocimientos técnicos y habilidades, así como la capacitación para el desempeño artesanal, artístico, recreacional y ocupacional, la protección y aprovechamiento de los recursos naturales y la participación ciudadana y comunitaria para el desarrollo de competencias laborales específicas.

1.3.2. Contribuir al proceso de formación integral y permanente de las personas complementando, actualizando y formando en aspectos académicos o laborales, mediante la oferta de programas flexibles y coherentes con las necesidades y expectativas de la persona, la sociedad, las demandas del mercado laboral, del sector productivo y las características de la cultura y el entorno.

A partir de lo que se expone en el Decreto 4904 de 2009, no resulta extraño, que dentro de los discursos naturalizados sobre lo educativo, el desarrollo humano esté supeditado al impacto significativo que tenga la educación en el desarrollo económico tal y como se señala en el numeral $1.2 \mathrm{y}$ en el inciso 1.3.1.

\section{Conclusiones}

El presente estudio, tuvo por prioridad, advertir si ¿era posible identificar un pensamiento educativo innovador, integrado a las necesidades de desarrollo humano en la región? Para dicho fín, y con el ánimo de poder establecer un punto de partida que puediera permitir una aproximación tanto explicativa como comprensiva de la cuestión eje de estudio, se optó por proponer una hipótesis de trabajo no explícita, que permitiera una aproximación más certera en la identificación de "un pesamiento educativo asociado a la innovación y a las necesidades de desarrollo humano de la región", así pues, se propuso, que dicha hipótesis, debía ser enunciada como sigue: de acuerdo al uso que cada comunidad e individuo dentro de una institución hace del concepto educación, ese uso, puede ser asociado eventualmente a unas categorías soportadas en enfoques teóricos, que harían evidente la naturalización o no de un tipo específico de pensamiento modelado o concepción adquirida sobre lo educativo manifiesto a través del discurso.

En consecuencia a la hipótesis de trabajo, lo primero que se optó, fue por identificar enfoques teóricos en estudios, trabajos de académicos, e informes que vincularan los conceptos educación y desarrollo, y que pudieran arrojar categorías genéricas sobre una educación para el desarrollo, y que permitiera una clasificación inicial para dar cuenta d elas categorías que serían utilizadas para el análisis de acuerdo a la frecuencia de su uso.

De dicha identificación, se obtuvó, que la educación para el desarrollo puede ser entendido desde dos dimensiones, como una educación para el crecimiento económico, en el que la productivada surge como un medio y un fin, soportado en un modelo educativo para el trabajo, y otro tipo de concepción sobre la educaicón para el desarrollo asociado a lo humano, en el cual, se supone que la finalidad de la educaicón para el desarrollo, es la formación de un criterio entre los invididuos, que pueda dar lugar a 
un pensamiento crítico, propositivo e integrado a las dinámicas sociales en pro del desarrollo y binestar de la comunidad.

Así pues, y de acuerdo al rastreo, se logró identificar un trabajo investigativo realizado en la universidad de Navarra Pamplona, por las doctoras Gabriela Orduna Allegrini y Concepción Naval Duran en el año 2001 titulado, "Educación para el desarrollo humano, educación como ayuda al crecimiento".

De esta investigación, se obtuvieron las categorías que sirvieron de soporte para caracterizar las dos miradas sobre una educación para el desarrollo.

De acuerdo a lo anterior, las categorías resultantes fueron las de aprendizaje social (AS) y educador social (ES). Categorías que de acuerdo a su definición, podrían contribuir a identificar la asociación de un concepto sobre una tendencia económica de educación para el desarrollo. Del mismo modo, se procedió para la dimensión de la educación asociada al desarrollo humano, de éste se hallaron dos categorías, la de acción educativa (AE) e inter educación (IE). Categorías que en su definición, se pueden asociar a un uso conceptual particualr sobre el desarrollo asociado a lo humano, y que además, puede contribuir a verificar en consonancia con las categorías asociadas a una educación para el desarrollo como crecimiento económico a la corroboración de la hipótesis de trabajo, la cual y de acuerdo a los resultados de los análisis obtenidos, resultaron positivas para la hipótesis; es decir, se pudo comprobar, que sí es posible identificar una frecuencia de uso significativa de las categorías asociadas a las tendecias de la educaciónb para el desarrollo desde sus dos dimensiones, una tendencia manifitesta en el pensamiento evidente en el uso discursivo sobre lo educativo en una población específica, para este caso, la muestra representativa del departamento de Caldas.

La corroboración de la hipótesis se logro gracias a un análsis denominado indice de frecuencia, ampliamente descrito, en el marco metodológico, y que dio lugar para identificar la frecuencia nominal de una de las dimensiones sobre el desarrollo en la que si bien, y pese a que la política desarrollista privilegia una mirada sobre lo educativo como un factor para la expansión del capital económico a través de la formación técnica y tecnológica, en lo relativo al departamento de Caldas, y tomando como fuente de referencia la mediana estadística obtenida, se puedo demostrar que existe una concepción naturalizada en el discurso sobre lo educativo, en el que se concibe a la educación, como un mecanismo para el mejoramiento humano y social de la comunidad, y no sólo como un medio o salida para la empleabili- dad, aspecto que de acuerdo Orduna y Naval (2011) mostrarían una clara tendencia por la dimensión del desarrollo asociado a lo humano.

En este sentido, y de acuerdo a Orduna y Naval (2011) "cuanto más crezca cada miembro de la comunidad como persona, más activamente podrá luchar contra la pobreza y a favor de su propio desarrollo y el de sus convecinos" (p. 2) postura, que la población encuestada adjudica a la educación como motor para el desarrollo social de la comunidad, pero que además, muestra un pensamiento sobre lo educativo romántico y asociado a la idea de una educación como posibilidad de emancipación y generación de civilidad y mejores posibilidades para lo humano y su entorno inmediato.

- Identificar la frecuencia nominal o uso conceptual de una determinada característica sobre el desarrollo en los discursos educativos regionales.

- Relacionar la frecuencia y uso nominativo de conceptos sobre el desarrollo y el desarrollo humano que abran nuevos comprensiones sobre la innovación como lenguaje del desarrollo humano.

En este sentido, y de acuerdo a lo que se puede extraer de los resultados obtenidos en el presente estudio, es que

Una segunda conclusión a la que se podría llegar de acuerdo a la validación y análisis de la información, es que pese a que es posible evidenciar a partir de un análisis estadístico el índice de frecuencia de una nominación naturalizada en el discurso de los estudiantes, docentes y directivos, acerca de lo educativo como una base fundamental para alcanzar niveles significativos de desarrollo personal y social en pro del bienestar de la comunidad entendido esto como un indicador de una educación para el desarrollo humano, esta tendencia, se hace dependiente del valor potencial que lo educativo representa como anclaje para salir de la pobreza relativa entendida ésta, como las diferencias generadas por los estándares de vida que se anticipan como condición y posibilidad deriva del hecho de ser escolarizado.

Lo anterior se corrobora, en lo que propone la política pública desde los objetivos del milenio oDM, y lo previsto en los informes del Consejo Privado de Competitividad CPC 2013 y 2014, para los cuales, el acceso al desarrollo humano será posible en la medida que se logre una concepción unánime de lo educativo como motor para el desarrollo económico. Aspecto, que por lo menos para el caso del depar- 
tamento Caldas parece de acuerdo a los análisis realizados páginas atrás, una constante claramente identificable.

No obstante, y pese que se ha corroborado lo que se anticipaba en la teoría sobre el desarrollo y su mirada de la educación como un vínculo estratégico de expansión del capital financiero y económico, quedan preguntas sin responder acerca, de ¿por qué si el sector educativo presenta una alta relación de institucionalización en términos de una concepción casi unánime sobre lo educativo desde la perspectiva del desarrollo económico, en los informes, programas y demás estudios sobre desarrollo y equidad social, prevalece la idea de atraso, falta de acceso y bienestar social, volviendo a candidatizar a la educación como salida a la problemática de descomposición social.

Así mismo, si el desarrollo económico depende en buena medida del impacto utilitario que puede tener la educación por lo menos como lo manifiestan los informes del CPC, OEI, Unesco, CEPAL, OMC, MEN, etc., y de allí derivar la posibilidad para que se dé el desarrollo humano, ¿no será, que se está asumiendo la incapacidad del sistema al nominar al sector educativo como la base para dar respuesta a problemas de mayor envergadura como la crisis de valores sociales y culturales, cuando el sistema mismo, está haciendo de lo educativo un simple medio de expansión de capital?

Sin embargo y antes de ir sobre las conclusiones, cabe advertir, que al hablar de pensamiento educativo innovador, se está aludiendo a la posibilidad que se tuvo de identificar en la percepción de los estudiantes sobre lo educativo, un entorno positivo y propicio para el aprendizaje colectivo en caminado a la construcción de comunidad como eje de bienestar social, basado en el intercambio de experiencias, y trabajo conjunto como base, para que se den los procesos de retroalimentación positiva entendida ésta, como los cambios que puedan resultar significativos en consonancia al conjunto de relaciones que se presentan y se generan desde la escuela como sistema, y otros agentes de la comunidad, y a partir de un medio propicio para la creación de incentivos y recursos que puedan dar lugar a la generación de nuevas innovaciones, siendo ese medio las interacciones o conjunto de relaciones como se describió más arriba entre escuela y otros agentes de la comunidad.

En este sentido, el pensamiento innovador podría ser tomado como la posibilidad de producir, asimilar y explotar con éxito la novedad en los ámbitos sociales y económicos. No obstante, lo que se presupone detrás del pensamiento innovador, es cierta suerte de incorporación de un nuevo método, proceso o estruc- tura que pueda dar un giro no sólo al sistema que lo contiene, sino al medio en el que éste se encuentra asociado apartir del mejoramiento o inclusión de otras lógicas de operatividad.

De acuerdo a esto, un pensamiento educativo innovador debería eventualmente desde la perspectiva que se abordo en el presente estudio, dar cuenta de una estrecha relación entre la tendencia económica del medio, y su asociación a los subcomponentes de aprendizaje social y educador social, como formas dialogadas del sistema educativo y de la comunidad en la que éste se encuentra articulado, de igual manera, pero en un sentido disimil, dar cuenta de la posibilidad de una educación que incluyera lo humano como base para la interacción social de los individuos más allá de la complejización del capital cognitivo, y a través, de la agenciación de una acción educativa clara y definida en un horizonte de oportunidades de formación no solo para la interacción productiva, sino también, para la transformación de su medio vital desde una puesta en escena del acto educativo como acción comunitaria.

\section{Limitaciones e investigaciones futuras}

Una limitación del presente estudio es del orden de la validez interna, ya que por tratarse de un diseño no experimental con datos estadísticos de percepción en el que se indagó por el uso/frecuencia de un determinado número de categorías asociadas a dos miradas del desarrollo, no se puede concluir que exista una relación causal directa entre el uso nominativo que prevalece sobre lo educativo en el departamento de Caldas, y el impacto positivo o no que la educación tenga en el desarrollo local de las comunidades objetos de estudio, ya que se dejaron de lado otras variables que podrían brindar luces acerca de la relación entre ambientes escolares y el desarrollo humano y social.

Al respecto, solo es posible afirmar que desde los hallazgos en el análisis de los datos, se muestra una clara fractura entre lo que se concibe como función social de la educación, y lo que la educación como estrategia del desarrollo realmente ha logrado a partir de los procesos de escolarización y expansión de posibilidades para el despliegue social del bienestar, la justicia y el acceso a las oportunidades tanto individuales como grupales de las comunidades indagadas. 


\section{Referencias bibliográficas}

Bourdieu, P. y Passeron, J.C. (2009). Los herederos. Los estudiantes y la cultura. Madrid: Siglo XXI.

Castoriadis, C. (1997). El imaginario social instituyente. Revista Zona erógena, 35. [En línea]. Recuperado el 28 de octubre de 2013 de: www.educ.ar.

Celorio, G. y López, A. (coord.) (2007). Diccionario de Educación para el Desarrollo. Bilbao: Hegoa.

Consejo Privado de Competitividad (CPC) (2013). Informe nacional de competitividad. Bogotá: Zetta Comunicadores.

Consejo Privado de Competitividad (CPC) (2014). Informe nacional de competitividad. Bogotá: Zetta Comunicadores.

De Sousa Santos, B. (2010). Para decolonizar Occidente: más allá del pensamiento abismal. Buenos Aires: Clacso.

Escobar, A. (1987). Power Visibility: Develoment and the Invention and Management of the Third World. [En línea]. Recuperado el 12 de octubre de 2013 de: http:// www.unc.edu/ aescobar/text/esp/arturoes.pdf

Escobar, A. (1999). El final del salvaje: naturaleza, cultura y política en la antropología contemporánea. Bogotá: Cerec.

Escobar, A. (2005). Más allá del tercer mundo, globalización y diferencia. Bogotá: Instituto Colombiano de Antropología e Historia.

Escobar, A. (2007). La invención del tercer mundo. Construcción y deconstrucción del desarrollo. Caracas: Fundación Editorial El Perro y La Rana.

Escobar, A. (2010). Una minga para el posdesarrollo. Venezuela: Universidad Nacional Mayor de Santa Cruz.

Flórez, P.T. (2012). Desarrollo y política educativa. Bogotá: Fundación Universitaria del Área Andina.

Fontalvo, R. (comp.) (2009). Complejidad y Ciencias Sociales y Humanas. Barranquilla: Ed. Universidad Simón Bolivar.

Granados, H. (2010). La educación tras la globalización, una mirada al desarrollo desde lo humano. Revista Filosófica y Literaria 6(6), 54-66.

Hernández, S., Fernández, C., Baptista, L. (2010). Metodología investigativa. México: Mc Graw Hill.

Hunter, I. (2000). Repensar la escuela: subjetividad, burocracia y crítica. Barcelona: Pomares-Corredor.

Inglehart, R. (1998). Modernización y posmodernización. El cambio cultural, económico y político en 43 sociedades. Madrid: Ed. Centro de Investigaciones Sociológicas.
Inglehart, R. y Welzel, C.H. (2006). Modernización, cambio cultural y democracia. La secuencia del desarrollo humano. Madrid: Siglo XxI editores.

Manucci, M. (2010). Contingencias. 5 Desafíos de cambio para una nueva década. Bogotá: Norma.

Marinis, de P. et al. (2010). La comunidad como pretexto. En torno al (re)surgimiento de las solidaridades comunitarias. México: Anthropos, Universidad Autónoma Metropolitana de México.

Martínez B., A. (2010). Verdades y mentiras sobre la escuela. Bogotá: Instituto para la Investigación Educativa y el Desarrollo Pedagógico (IDEP).

Martínez B., A. et al. (2009). Los bordes de la pedagogía: del modelo a la ruptura. Bogotá: Universidad Pedagógica Nacional.

Martínez B., A. et al. (1994). Currículo y Modernización. Cuatro décadas de educación en Colombia. Bogotá: Foro Nacional por Colombia y Tercer Milenio.

Meirieu, P. (2004). En la escuela hoy. Barcelona: Octaedro.

Michéa, J.C. (2009). "La escuela de la ignorancia” y sus condiciones modernas. Madrid: Acuarela y Machado.

Ministerio de Educación Nacional (MEN) (2001). Informe nacional sobre el desarrollo de la educación en Colombia. Bogotá.

Ministerio de Educación Nacional (MEN) (2007). Investigación de los saberes pedagógicos. Bogotá.

Ministerio de Educacional (MEN), (2009). Decreto N-4904. Por el cual se reglamenta la organización, oferta y funcionamiento de la prestación del servicio educativo para el trabajo y el desarrollo humano y se dictan otras disposiciones. Bogotá.

Monbeig, P. (2009). Cahiers des ameriques latines. Philosophie de la libération et tourantdécolonial. Sorbone. Paris III France.

Moro, B. (2010). Cátedras del milenio. Objetivos del Desarrollo del Milenio.

Negri, T. et al. (2008). Imperio, multitud y sociedad abigarrada. La Paz: Clacso.

Ohmae, K. (2005). El próximo escenario global. Desafíos y oportunidades en un mundo sin fronteras. Bogotá: Norma.

Oppenheimer, A. (2011). "Basta de historias". La obsesión latinoamericana con el pasado y las 12 claves del futuro. Bogotá: Debate.

Paquay, L. et al. (2005). La formación profesional del maestro. Estrategias y competencias. México: Fondo de Cultura Económica. 
Popkewitz, T. (1988). Paradigma e ideología en la investigación educativa. Madrid: Mondadori.

Popkewitzt, T. et al. (2007). El mito de las reformas educativas. Un estudio de las respuestas de la escuela ante un programa de cambio. Barcelona: Pomares-Corredor.

Quijano, A. (2000). El fantasma del desarrollo. Revista Venezolana de Economía y Ciencias Sociales, 6 (2), 73-90.

Said, E. (2008). Orientalismo. Barcelona: Debolsillo.

Schaeffer, J.M. (2009). El fin de la excepción humana. Buenos Aires: Fondo de Cultura Económica.

Serna, A.E. (2004). El pedagogo y el político. Bogotá: Universidad Distrital.

Solanas, A, Salafranca, L, Fauquet, J, Nuñez, M. (2005). Estadística descriptiva en ciencias del comportamiento. Madrid: Thomson International y Paraninfo.
Terren, E. (1999). Educación y modernidad. Entre la utopía y la burocracia. Madrid: Anthropos y Universidad de la Coriña.

Unesco (2004). El desarrollo de la educación en el siglo XXI. Informe nacional de Colombia. Bogotá: Unesco.

Unesco (2013). Informe de seguimiento de la EPT en el mundo. Aprender y enseñar para el desarrollo. Paris: Unesco.

Vilar, P. (1993). Crecimiento y desarrollo. Barcelona: Planeta Agostini.

Zuluaga, O. et al. (2003). Pedagogía y epistemología. Bogotá: Magisterio. 